\title{
Fatores de risco para doenças crônicas em agentes comunitários de saúde de um município do interior de Minas Gerais, Brasil
}

\author{
Risk factors for chronic diseases in community health workers \\ in a municipality in the countryside of Minas Gerais, Brazil
}

\section{Factores de riesgo para enfermedades crónicas en trabajadores de salud comunitarios en un municipio del interior de Minas Gerais - Brasil}

\author{
1Prefeitura Municipal de Poços de Caldas - Poços de Caldas (MG), Brasil. \\ 2Universidade Federal de Lavras - Lavras (MG), Brasil. \\ ${ }^{3}$ Instituto Brasileiro de Geografia e Estatística - Varginha (MG), Brasil. \\ ${ }^{4}$ Sapienza Universitá di Roma - Roma, Itália. \\ 5Universidade Federal de São Paulo - São Paulo (SP), Brasil.
}

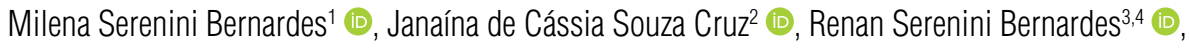

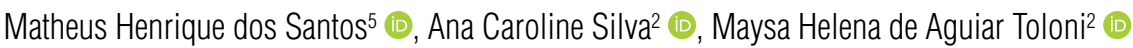

\section{Resumo}

Introdução: Estima-se que as doenças crônicas não transmissíveis (DCNT) e seus agravos sejam responsáveis por aproximadamente $70 \%$ das mortes no mundo. A falta de prevenção e de gerenciamento adequado dessas patologias acaba demandando assistência médica de custos crescentes, em função da permanente incorporação tecnológica. No que diz respeito à saúde do trabalhador, o aumento da prevalência dos casos de DCNT pode resultar em absenteísmo e invalidez e repercutir na qualidade do trabalho ofertado. Objetivo: Avaliar a presença dos fatores de risco para DCNT em agentes comunitários de saúde (ACS). Métodos: Trata-se de estudo transversal desenvolvido em município do estado de Minas Gerais. Foram entrevistados 139 ACS, que responderam a um questionário com perguntas fechadas e pré-categorizadas. Foram aferidos a circunferência da cintura, o peso e a estatura, e foram calculados o índice de massa corporal (IMC), o índice de conicidade e a relação cintura/estatura. Resultados: O excesso de peso esteve presente em $56,1 \%$ dos entrevistados, dos quais $30,2 \%$ eram obesos. Ademais, $51,8 \%$ dos ACS foram classificados como sedentários, e 14,4\% relataram ser fumantes. O risco elevado de desenvolver doença cardiovascular foi observado em $27,27 \%$ dos ACS do sexo masculino e em $57,81 \%$ dos do sexo feminino. O consumo de pelo menos um alimento ultraprocessado foi relatado por $53,9 \%$ dos ACS, e observou-se associação positiva entre o consumo desses alimentos com o estado nutricional $(p=0,032)$. Conclusões: Os resultados mostram significativa prevalência de fatores de risco de DCNT entre os ACS. Considerando-se o impacto dessas doenças para a saúde e a qualidade do trabalho, é fundamental que a vigilância e a prevenção dos fatores de risco estejam presentes na programação de saúde dos municípios.

Palavras-chave: Doenças crônicas. Saúde do trabalhador. Atenção primária à saúde. Agentes comunitários de saúde.

Como citar: Bernardes MS, Cruz JCS, Bernardes RS, Santos MH, Silva AC, Toloni MHA. Fatores de risco para doenças crônicas em agentes comunitários de saúde de um município do interior de Minas Gerais, Brasil. Rev Bras Med Fam Comunidade. 2021;16(43):2661. https://doi.org/10.5712/rbmfc16(43)2661

\author{
Autor correspondente: \\ Milena Serenini Bernardes \\ E-mail: miserenini@gmail.com \\ Fonte de financiamento: \\ não se aplica. \\ Parecer CEP: \\ CAAE 80673417.8.0000.5148 \\ Procedência: \\ não encomendado. \\ Avaliação por pares: \\ externa. \\ Recebido em: 04/08/2020. \\ Aprovado em: 15/09/2021. \\ Editora Associada: \\ Márcia Silveira Ney
}




\begin{abstract}
Introduction: chronic non-communicable diseases (CNCDs) and their injuries are estimated to be responsible for approximately $70 \%$ of deaths worldwide. The lack of prevention and good management of these pathologies leads to the need for medical assistance with increasing costs, due to the permanent technological incorporation. With regard to the occupational health, the increase in prevalence of cases of CNCDs can result in absenteeism, disability and impact on the quality of the work offered. Objective: to evaluate the presence of risk factors for CNCDs in community health workers (CHW). Methods: This is a cross-sectional study developed with $\mathrm{CHWs}$ from a municipality in the state of Minas Gerais. Results: $139 \mathrm{CHWs}$ of both sexes, belonging to the 36 family health units of the municipality, were interviewed by means of a questionnaire with closed and pre-categorized questions on demographic and socioeconomic data, food consumption and health characteristics. Waist circumference (WC), weight and height were measured, and BMI, conicity index and waist-to-height ratio were calculated. A prevalence of married females and white workers was pointed. Overweight was present in $56.1 \%$ of respondents. In addition, $51.8 \%$ of CHWs were classified as sedentary, and $14.4 \%$ reported being smokers. The high risk of developing cardiovascular diseases was stated in $27.27 \%$ of male $\mathrm{CHWs}$ and $57.81 \%$ of female $\mathrm{CHWs}$. Consumption of at least one ultra-processed food was reported by $53.9 \%$ of respondents, and a positive association between the consumption of these foods-including sandwiches, pizzas and chips—and the nutritional status of the CHWs was pointed. Conclusions: The results show a high prevalence of risk factors for CNCDs among CHWs. Considering the impact of CNCDs on health and quality of work, surveillance and prevention of risk factors, also aimed at workers' health, are present in the health programming of municipalities.
\end{abstract}

Keywords: Chronic diseases. Occupational health. Primary health care. Community health workers.

\title{
Resumen
}

Introducción: Se estima que las enfermedades crónicas no transmisibles (ENT) y sus lesiones son responsables de aproximadamente el 70\% de las muertes en todo el mundo. La falta de prevención y buen manejo de estas patologías acaba exigiendo asistencia médica con costos crecientes, debido a la permanente incorporación tecnológica. Con respecto a la salud de los trabajadores, el aumento en la prevalencia de casos de ENT puede resultar en ausentismo, discapacidad e impacto en la calidad del trabajo ofrecido. Objetivo: Evaluar la presencia de factores de riesgo de enfermedades crónicas no transmisibles (ENT) en los agentes comunitarios de salud comunitarios (ACS). Métodos: Este es un estudio transversal desarrollado en un municipio del estado de Minas Gerais. Se entrevistaron 139 ACS, quienes respondieron un cuestionario con preguntas cerradas y pre-categorizadas. Se midieron la circunferencia de la cintura, el peso, la altura y se calcularon el IMC, el índice de inclinación y la relación cintura / altura. Resultados: El exceso de peso estuvo presente en el $56,1 \%$ de los encuestados. Además, el $51,8 \%$ de los ACS se clasificó como sedentario, y el 14,4\% informó ser fumador. El alto riesgo de desarrollar enfermedad cardiovascular se observó en el $27,27 \%$ de los ACS masculinos y en el $57,81 \%$ de los ACS femeninos. El 53,9\% del ACS informó el consumo de al menos un alimento ultraprocesado, y se observó una asociación positiva entre el consumo de estos alimentos y el estado nutricional. Conclusiones: Los resultados muestran una alta prevalencia de factores de riesgo de ENT entre ACS. Considerando el impacto de estas enfermedades en la salud y la calidad del trabajo, es esencial que la vigilancia y prevención de los factores de riesgo estén presentes en la programación de salud de los municipios.

Palabras-clave: Enfermedad crónica. Salud laboral. Atención primaria de salud. Agentes comunitarios de salud.

\section{INTRODUÇÃO}

A Atenção Primária à Saúde (APS) tem sido alvo de interesse e prioridade das políticas públicas nas últimas décadas no Brasil e no mundo. De modo geral, as propostas de reforma dos sistemas de saúde atribuem à atenção primária o papel de porta de entrada e de coordenação do conjunto de ações e serviços dos demais níveis do sistema de saúde, caracterizando-a como o locus de resolubilidade da maior parte dos problemas de saúde presentes em uma dada população por meio da atenção prestada aos pacientes, famílias e comunidades no decorrer do tempo. ${ }^{1}$

A profissão do agente comunitário de saúde (ACS) foi legalmente reconhecida em 2002 pela Lei 10.507, embora o Programa Nacional de Agentes Comunitários de Saúde (PACS) tenha sido criado no início da década de 90 . Atualmente existem mais de 250 mil ACS no Brasil, e esses profissionais configuram-se como elo de fortalecimento e ligação entre a atenção primária e a comunidade, exercendo ainda funções de cadastramento das famílias adscritas às áreas das equipes de Saúde da Família, das visitas domiciliares, e o desenvolvimento de ações de educação, prevenção de doenças, promoção da saúde e vigilância em saúde. ${ }^{2-4}$ 
Nota-se crescente interesse em pesquisas relacionadas aos processos de trabalho e seus impactos sobre a saúde do trabalhador. Sabe-se que as cargas de trabalho podem atuar sobre o corpo do indivíduo e demandam processos de adaptação que podem resultar em danos físicos e psíquicos. ${ }^{5}$ Estudos têm avaliado a penosidade relacionada ao trabalho dos ACS, os quais rotineiramente caminham longos percursos, algumas vezes sob condições climáticas desfavoráveis, trabalham em áreas de risco, visitam locais insalubres e presenciam situações de violência e vulnerabilidade nas famílias que acompanham. Essas condições de trabalho podem elevar o risco de acidentes e de doenças, impactando diretamente o trabalho desempenhado e a saúde do trabalhador. ${ }^{6,7}$

Ainda são escassos os trabalhos que se dedicam a avaliar o perfil de saúde e os fatores de risco para doenças crônicas não transmissíveis (DCNT) entre ACS. Sabe-se que a realidade epidemiológica, social e demográfica atual tem influenciado negativamente na saúde da população, aumentando o risco de desenvolvimento de excesso de peso e DCNT. ${ }^{8}$ Dessa forma, é de fundamental importância que se identifique o nível de estresse físico e mental dos ACS para que seja possível elaborar ações voltadas para a melhora da qualidade de vida e das condições de trabalho que têm gerado sofrimento e agravos à saúde desses trabalhadores.

O levantamento dos fatores de risco presentes nos ACS que podem levar ao surgimento de DCNT, tais como inatividade física, obesidade, baixa qualidade da alimentação e tabagismo, também é importante ponto de investigação para que se possa atuar na qualidade de vida e do trabalho desses profissionais. Diante desse contexto, o objetivo do presente estudo foi avaliar a presença dos fatores de risco para DCNT nessa população.

\section{MÉTODOS}

\section{Desenho do estudo e universo Amostral}

Trata-se de um estudo transversal desenvolvido com ACS do município de Poços de Caldas, no estado de Minas Gerais. A população estimada do município é de 168.641 habitantes. ${ }^{8}$ No que diz respeito à atenção primária, o município possui 35 equipes de Saúde da Família, e a estimativa da população coberta pela Estratégia Saúde da Família (ESF) é de 72,13\%. Em dezembro de 2020 o número total de ACS foi de 185 , cobrindo $64,04 \%$ da população. ${ }^{9}$

O desenho metodológico incialmente proposto para este estudo pretendia entrevistar a totalidade dos ACS do município. No entanto, a amostra final foi composta de 139 ACS, de ambos os sexos, de todas as 35 equipes de Saúde da Família, o que corresponde a 75,13\%. Foram excluídos da pesquisa os ACS que estavam de férias, de licença e em afastamento e aqueles que se recusaram a participar do estudo.

\section{Coleta de dados}

Os participantes da pesquisa responderam a um questionário com perguntas fechadas e précategorizadas sobre características demográficas, socioeconômicas, de saúde, nível de atividade física e consumo alimentar. Foram utilizados instrumentos validados pela literatura científica. Os questionários utilizados foram previamente testados com dez participantes com os objetivos de avaliação, revisão e aprimoramento. A coleta de dados aconteceu de março a agosto de 2018. 
As entrevistas e a aferição de dados antropométricos foram realizadas in loco nas ESF por equipe devidamente treinada. Com o intuito de garantir a qualidade dos dados obtidos, foi ainda construído um manual de campo para orientar os entrevistadores durante essa etapa do trabalho, informando sobre a forma de aplicar o questionário e de aferir as medidas antropométricas do estudo.

\section{Variáveis da pesquisa}

\section{Perfil socioeconômico e de saúde}

A fim de obter informações relacionadas a perfil socioeconômico, composição familiar, perfil demográfico e de saúde, foram analisadas as seguintes variáveis: idade do respondente; sexo; cor (branca, preta, pardo, amarela); estado civil (casado, união estável, solteiro, divorciado); nível de escolaridade (ensino fundamental completo, ensino médio incompleto, ensino médio completo, superior incompleto, superior completo); autopercepção de estado (muito bom, bom, regular, ruim, muito ruim, não sabe); tabagismo (nunca fumou; fumou, mas parou; fuma até cinco cigarros/dia; fuma de cinco a dez cigarros/ dia; fuma de dez a 20 cigarros/dia; fuma mais de 20 cigarros/dia).

\section{Avaliação antropométrica}

O peso foi aferido por balança de escala mecânica da marca Filizola ${ }^{\circledR}$ com capacidade para $150 \mathrm{~kg}$ e precisão de $100 \mathrm{~g}$, sobre a qual o participante se posicionou ereto, com roupas leves, descalço e no centro. Foi realizada a calibragem da balança antes da aferição. A altura foi medida em estadiômetro afixado na própria balança, com o participante em pé, descalço, com o corpo ereto e olhando para a frente. Com as variáveis de peso e altura foram calculados índice de massa corporal (IMC) e peso (kg)/ altura $^{2}(\mathrm{~m})$, e foi utilizada a classificação de IMC de acordo com os pontos de corte recomendados pela Organização Mundial da Saúde (OMS). ${ }^{9,10}$

A circunferência da cintura (CC) foi aferida com o participante em posição ereta, no ponto médio entre a extremidade da última costela e a crista ilíaca. Foi utilizada fita antropométrica inelástica, de $150 \mathrm{~cm}$ de comprimento e precisão de uma casa decimal. Solicitou-se que o participante respirasse normalmente no momento da medida. Foram adotados os pontos de corte para CC da OMS, ${ }^{11}$ considerando-se CC >80 e $94 \mathrm{~cm}$ como risco para o desenvolvimento de doenças cardiovasculares e CC >88 e $102 \mathrm{~cm}$ como risco elevado para o desenvolvimento de doenças cardiovasculares, para mulheres e homens, respectivamente.

O índice de conicidade foi calculado, e estabeleceram-se os pontos de corte 1,26 para homens e 1,18 para mulheres. ${ }^{12}$ A relação cintura/estatura (RCEst) também foi calculada, com 0,52 e 0,53 como pontos de corte para homens e mulheres, respectivamente. ${ }^{13}$

\section{Consumo alimentar}

A ingestão alimentar foi estimada utilizando-se questionário adaptado do Sistema de Vigilância Alimentar e Nutricional (SISVAN). ${ }^{14}$ Os participantes foram questionados sobre o consumo de alimentos no dia anterior à entrevista. Optou-se pele emprego de questionário adaptado do SISVAN por este já ser o instrumento utilizado no âmbito da APS para a avaliação do consumo alimentar. O questionário avaliou 
o consumo de alimentos in natura ou minimamente processados, o consumo de alimentos processados e alimentos ultraprocessados (AUP). Também foi avaliado o hábito de assistir à televisão enquanto se realizam as refeições.

\section{Nível de atividade física}

O nível de atividade física foi estimado pelo Questionário Internacional de Atividade Física (IPAQ). ${ }^{15}$ Foi utilizado apenas o módulo de lazer do IPAQ. Os indivíduos que relataram praticar atividade física por pelo menos 150 minutos por semana, ou 75 minutos de atividade física vigorosa, foram classificados como ativos. Indivíduos com prática de atividade física semanal superior a 10 minutos mas inferior a 150 minutos de atividade moderada ou 75 minutos de atividade física vigorosa foram classificados como insuficientemente ativos. E aqueles que praticavam menos de 10 minutos de atividade física por semana foram classificados como inativos/sedentários.

\section{Análise dos dados}

Os dados coletados foram analisados pelo programa $R$ i386, versão 3.4.4. Foi efetuada análise exploratória por meio do cálculo de frequências simples e relativas, e estimou-se o intervalo de confiança de 95\% (IC95\%) das variáveis selecionadas. Para as variáveis contínuas foi realizado cálculo de média, desvio padrão (DP), valores mínimo e máximo. A normalidade da distribuição foi verificada utilizando-se o teste de Kolmogorov-Smirnov. Aplicou-se o teste qui-quadrado de Pearson com nível de significância de $5 \%(p<0,05)$ para avaliar a associação entre as variáveis.

\section{Aspectos éticos}

Este trabalho faz parte do projeto de pesquisa intitulado "Modos de Vida de Agentes Comunitários de Saúde de um Município do Interior de Minas Gerais (MG)", aprovado pelo Comitê de Ética em Pesquisa da Universidade Federal de Lavras sob o parecer $n^{\circ} 2.442 .225$. Todos os participantes foram informados sobre os objetivos da pesquisa, e aqueles que concordaram assinaram o termo de consentimento livre e esclarecido (TCLE) em duas vias.

\section{RESULTADOS}

Dos 139 ACS entrevistados, 92,1\% são do sexo feminino, 58,2\% são casados, e a média de idade foi de 37,2 anos (DP 7,6 anos). Em relação ao nível de escolaridade, 30,9\% não possuem nível médio completo, mas já exerciam suas funções quando foi sancionada a Lei no 13595/2018, que altera a escolaridade mínima para a função de ACS. No que diz respeito à cor, 55,4\% declararam-se de cor branca (Tabela 1). A avaliação das características de saúde e dos hábitos de vida dos ACS demonstrou que a maior parte dos participantes avaliam positivamente seu estado atual de saúde; $27,3 \%$ consideram-no como "muito bom" e 51,1\% como "bom". Mais da metade dos ACS foram classificados como sedentários. Além disso, 55,8\% relataram assistir à televisão enquanto realizam as refeições. Em relação ao hábito de fumar, $14,4 \%$ são fumantes e 1,4\% relataram consumo superior a 20 cigarros por dia (Tabela 2). A avaliação do estado nutricional demonstrou que $43,9 \%$ são eutróficos, $25,8 \%$ foram classificados 
Tabela 1. Características sociodemográficas de agentes comunitários de saúde. Poços de Caldas (MG), 2018.

\begin{tabular}{|c|c|c|c|c|}
\hline \multirow{2}{*}{ Variáveis } & \multirow{2}{*}{$\mathbf{n}$} & \multirow{2}{*}{$\%$} & \multicolumn{2}{|c|}{ IC95\% } \\
\hline & & & LI & LS \\
\hline \multicolumn{5}{|l|}{ Idade (anos) } \\
\hline Até 30 & 29 & 21,0 & 17,9 & 24,0 \\
\hline 31 a 40 & 59 & 42,7 & 39,0 & 46,4 \\
\hline 41 a 50 & 38 & 27,5 & 24,2 & 30,8 \\
\hline 51 a 60 & 11 & 7,9 & 5,9 & 10,0 \\
\hline Mais de 60 & 01 & 0,7 & 0,0 & 1,3 \\
\hline \multicolumn{5}{|l|}{ Sexo } \\
\hline Masculino & 11 & 7,9 & 5,8 & 9,9 \\
\hline Feminino & 128 & 92,0 & 90,0 & 94,1 \\
\hline \multicolumn{5}{|l|}{ Estado civil } \\
\hline Solteiro & 35 & 25,1 & 21,9 & 28,4 \\
\hline União estável & 10 & 7,1 & 5,2 & 9,1 \\
\hline Casado & 81 & 58,2 & 54,5 & 61,9 \\
\hline Divorciado & 12 & 8,6 & 6,5 & 10,7 \\
\hline \multicolumn{5}{|l|}{ Cor ou raça } \\
\hline Branca & 77 & 55,4 & 51,6 & 59,1 \\
\hline Preta & 18 & 12,9 & 10,4 & 15,4 \\
\hline Parda & 40 & 28,7 & 25,3 & 32,1 \\
\hline Amarela & 4 & 2,8 & 1,6 & 4,1 \\
\hline \multicolumn{5}{|l|}{ Escolaridade } \\
\hline Fundamental completo & 3 & 2,1 & 1,0 & 3,2 \\
\hline Médio incompleto & 8 & 5,7 & 4,0 & 7,5 \\
\hline Médio completo & 82 & 58,9 & 55,3 & 62,6 \\
\hline Superior incompleto & 22 & 15,8 & 13,1 & 18,5 \\
\hline Superior completo & 24 & 17,2 & 14,4 & 20,0 \\
\hline
\end{tabular}

IC: intervalo de confiança; LI: limite inferior; LS: limite superior.

Tabela 2. Características de saúde e hábitos de vida de agentes comunitários de saúde. Poços de Caldas (MG), 2018.

\begin{tabular}{|c|c|c|c|c|}
\hline \multirow{2}{*}{ Variáveis } & \multirow{2}{*}{$\mathbf{n}$} & \multirow{2}{*}{$\%$} & \multicolumn{2}{|c|}{ IC95\% } \\
\hline & & & LI & LS \\
\hline \multicolumn{5}{|c|}{ Como considera o estado de saúde } \\
\hline Muito bom & 38 & 27,3 & 24,0 & 30,6 \\
\hline Bom & 71 & 51,0 & 47,3 & 54,8 \\
\hline Regular & 24 & 17,2 & 14,4 & 20,0 \\
\hline Ruim/Muito ruim/Não sabe & 6 & 4,3 & 2,8 & 5,8 \\
\hline \multicolumn{5}{|l|}{ Tabagismo } \\
\hline Nunca fumou & 90 & 64,7 & 61,1 & 68,3 \\
\hline Fumou, mas parou & 29 & 20,8 & 17,8 & 23,9 \\
\hline
\end{tabular}


Tabela 2. Continuação.

\begin{tabular}{|c|c|c|c|c|}
\hline \multirow{2}{*}{ Variáveis } & \multirow{2}{*}{$\mathbf{n}$} & \multirow{2}{*}{$\%$} & \multicolumn{2}{|c|}{ IC95\% } \\
\hline & & & LI & LS \\
\hline Até 5 cigarros por dia & 8 & 5,7 & 4,0 & 7,5 \\
\hline 5 a 10 cigarros por dia & 4 & 2,8 & 1,6 & 4,1 \\
\hline 10 a 20 cigarros por dia & 6 & 4,3 & 2,8 & 5,8 \\
\hline Mais de 20 cigarros por dia & 2 & 1,4 & 0,5 & 2,3 \\
\hline \multicolumn{5}{|l|}{ Nível de atividade física } \\
\hline Sedentário & 72 & 51,8 & 48,0 & 55,5 \\
\hline Insuficientemente ativo & 19 & 13,6 & 11,1 & 16,2 \\
\hline Ativo & 48 & 34,5 & 30,9 & 38,0 \\
\hline \multicolumn{5}{|l|}{ Realiza as refeições vendo TV } \\
\hline Sim & 77 & 55,8 & 52,0 & 59,5 \\
\hline Não & 61 & 44,2 & 40,4 & 47,9 \\
\hline
\end{tabular}

IC: intervalo de confiança; LI: limite inferior; LS: limite superior.

como com sobrepeso e 30,2\% com obesidade. O IMC médio da população estudada foi de $28,1 \mathrm{~kg} / \mathrm{m}^{2}$ (Tabela 3). Entre as mulheres, 21,8\% apresentaram risco de desenvolver doenças cardiovasculares, com valores de CC maior do que $80 \mathrm{~cm}$, e 57,8\% apresentam risco elevado, com CC acima de $88 \mathrm{~cm}$. Já entre os ACS do sexo masculino, 18,2\% apresentaram risco e 27,2\% apresentaram risco elevado. Os indicadores de adiposidade abdominal mostraram-se elevados entre a maioria dos ACS. Foi observado índice de conicidade elevado entre $75,3 \%$ dos ACS, e 61,8\% apresentaram relação cintura/estatura acima do ponto de corte estabelecido.

Tabela 3. Indicadores antropométricos de agentes comunitários de saúde. Poços de Caldas (MG), 2018.

\begin{tabular}{lcc}
\hline Indicador antropométrico & Média/DP & Valor mín-máx \\
\hline IMC $\left(\mathrm{kg} / \mathrm{m}^{2}\right)$ & $28,16 / 4,9$ & $20,2-42,6$ \\
Circunferência da cintura $(\mathrm{cm})$ & $91,44 / 12,03$ & $67,4-128,8$ \\
Índice de conicidade & $1,40 / 0,085$ & $0,93-1,45$ \\
Relação cintura/estatura & $0,58 / 0,062$ & $0,47-0,77$ \\
\hline
\end{tabular}

DP: desvio padrão; Valor mín-máx: valor mínimo-valor máximo; IMC: índice de massa corporal.

Em relação ao consumo de alimentos saudáveis no dia anterior à entrevista, $74,6 \%$ dos entrevistados relataram ter consumido feijão, e o consumo de frutas, verduras de folha e hortaliças foi reportado por $73,1,57,2$ e 64,4\%, respectivamente (Tabela 4). O consumo de pelo menos um AUP (bebidas adoçadas; hambúrguer e/ou embutidos) no dia anterior à entrevista foi relatado por 53,9\% dos ACS. Foi observada associação positiva entre o consumo de AUP e de alimentos como sanduíches, pizza e batata frita com o estado nutricional dos ACS (Tabela 5). 
Tabela 4. Consumo alimentar de agentes comunitários de saúde no dia anterior. Poços de Caldas (MG), 2018.

\begin{tabular}{|c|c|c|c|c|}
\hline \multirow{2}{*}{ Variáveis } & \multirow{2}{*}{$\mathbf{n}$} & \multirow{2}{*}{$\%$} & \multicolumn{2}{|c|}{ IC95\% } \\
\hline & & & LI & LS \\
\hline \multicolumn{5}{|l|}{ Feijão } \\
\hline Sim & 103 & 74,64 & 71,36 & 77,91 \\
\hline Não & 35 & 25,36 & 22,09 & 28,64 \\
\hline \multicolumn{5}{|l|}{ Frutas } \\
\hline Sim & 101 & 73,19 & 69,85 & 76,52 \\
\hline Não & 37 & 26,81 & 23,48 & 30,15 \\
\hline \multicolumn{5}{|c|}{ Verduras de folha } \\
\hline Sim & 79 & 57,25 & 53,52 & 60,97 \\
\hline Não & 59 & 42,75 & 39,03 & 46,48 \\
\hline \multicolumn{5}{|l|}{ Legumes } \\
\hline Sim & 89 & 64,49 & 60,89 & 68,09 \\
\hline Não & 49 & 35,51 & 31,91 & 39,11 \\
\hline \multicolumn{5}{|c|}{ Hambúrguer e/ou embutidos } \\
\hline Sim & 25 & 18,12 & 15,22 & 21,01 \\
\hline Não & 113 & 81,88 & 78,99 & 84,78 \\
\hline \multicolumn{5}{|c|}{ Batata frita, pizza, sanduíches } \\
\hline Sim & 30 & 21,74 & 18,63 & 24,84 \\
\hline Não & 108 & 78,26 & 75,16 & 81,37 \\
\hline \multicolumn{5}{|c|}{ Bebidas adoçadas } \\
\hline $\operatorname{Sim}$ & 57 & 41,30 & 37,60 & 45,01 \\
\hline Não & 81 & 58,70 & 54,99 & 62,40 \\
\hline
\end{tabular}

IC: intervalo de confiança; LI: limite inferior; LS: limite superior.

Tabela 5. Associação entre estado nutricional e consumo alimentar de agentes comunitários de saúde. Poços de Caldas (MG), 2018.

\section{Estado nutricional}

\begin{tabular}{|c|c|c|c|c|c|c|c|c|c|}
\hline Variável & \multicolumn{2}{|c|}{ Normal } & \multicolumn{2}{|c|}{ Sobrepeso } & \multicolumn{2}{|c|}{ Obeso } & \multicolumn{2}{|c|}{ Total } & Valor $\mathrm{p}$ \\
\hline \multicolumn{10}{|c|}{ Ultraprocessados (bebidas adoçadas e/ou hambúrguer/embutidos) } \\
\hline Não & 17 & 32,1 & 27 & 50,9 & 9 & 17,0 & 53 & 38,4 & 0,032 \\
\hline \multicolumn{10}{|c|}{ Batata frita, pizza, sanduíche } \\
\hline Não & 30 & 27,8 & 52 & 48,1 & 26 & 24,1 & 108 & 78,3 & 0,022 \\
\hline \multicolumn{10}{|l|}{ Frutas } \\
\hline $\operatorname{Sim}$ & 27 & 26,7 & 45 & 44,55 & 29 & 28,7 & 101 & 73,1 & \multirow{2}{*}{0,811} \\
\hline Não & 8 & 21,6 & 17 & 45,9 & 12 & 32,4 & 37 & 26,8 & \\
\hline
\end{tabular}


Tabela 5. Continuação.

\begin{tabular}{|c|c|c|c|c|c|c|c|c|c|}
\hline \multicolumn{10}{|c|}{ Estado nutricional } \\
\hline \multirow{2}{*}{ Variável } & \multicolumn{2}{|c|}{ Normal } & \multicolumn{2}{|c|}{ Sobrepeso } & \multicolumn{2}{|c|}{ Obeso } & \multicolumn{2}{|c|}{ Total } & \multirow{2}{*}{ Valor $p$} \\
\hline & $\mathbf{n}$ & $\%$ & $\mathbf{n}$ & $\%$ & $\mathbf{n}$ & $\%$ & $\mathbf{n}$ & $\%$ & \\
\hline \multicolumn{10}{|l|}{ Legumes } \\
\hline Sim & 22 & 27,8 & 34 & 43,0 & 23 & 29,1 & 79 & 57,2 & \multirow{2}{*}{0,733} \\
\hline Não & 13 & 22,0 & 28 & 47,5 & 18 & 30,5 & 59 & 42,8 & \\
\hline \multicolumn{10}{|l|}{ Feijão } \\
\hline Sim & 27 & 26,7 & 45 & 44,5 & 29 & 28,7 & 101 & 73,2 & \multirow{2}{*}{0,142} \\
\hline Não & 8 & 21,6 & 17 & 45,9 & 12 & 32,4 & 37 & 26,8 & \\
\hline
\end{tabular}

Teste qui-quadrado. Foi considerado o valor de $p<0,05$.

\section{DISCUSSÃO}

Os resultados deste artigo apontam para a prevalência de excesso de peso em mais da metade dos ACS entrevistados, e a obesidade fez-se presente em $30,2 \%$. No que se refere aos fatores de risco para DCNT, 51,8\% não realizam atividade física, $14,4 \%$ são fumantes e o consumo de AUP no dia anterior à entrevista foi relatado por $53,9 \%$.

No tocante ao perfil sociodemográfico, assim como no presente trabalho, o estudo nacional que avaliou o perfil dos ACS no Brasil em 2015 já havia demonstrado prevalência de $83,2 \%$ do sexo feminino nessa categoria profissional. A maior presença de mulheres nas diversas profissões ligadas à área da saúde já é bem conhecida, e, especificamente em relação aos ACS, alguns autores relacionam esse processo de feminização à resistência da própria comunidade ao sexo masculino. Isso porque os ACS precisam de acesso às residências, e existiria maior facilidade da população em revelar suas particularidades para mulheres do que para homens. ${ }^{16,17}$

Em relação à percepção das condições de saúde, os resultados indicaram que a maioria dos ACS entrevistados avaliou positivamente seu estado atual de saúde. Dados nacionais observaram que 71,9\% dos ACS consideram ter boa saúde. ${ }^{18}$

Em relação à alta prevalência de sedentarismo, Colombo e Derquin, ${ }^{19}$ em estudo que avaliou o estado nutricional e o nível de atividade física nesses profissionais, observaram que $73 \%$ eram sedentários e 51,5\% apresentaram algum grau de sobrepeso ou obesidade. Os dados do nosso estudo aproximamse dos achados de pesquisa de base nacional com ACS, que observou que $52,9 \%$ costumam realizar atividade física no tempo livre. ${ }^{18}$ Embora o sedentarismo seja um fator de risco relevante para DCNT, ele pode ser modificado e deve ser estimulado por políticas públicas voltadas para a promoção da atividade física. ${ }^{20}$

No que diz respeito aos hábitos de vida, mais da metade dos ACS entrevistados relataram assistir à televisão concomitantemente à realização das refeições. Um estudo conduzido por Maia et al. ${ }^{21}$ indicou que o hábito de assistir à televisão está relacionado ao maior consumo de alimentos não saudáveis em detrimento dos considerados saudáveis. Essa condição foi observada em ambos os sexos, independentemente da faixa etária e do nível de escolaridade do indivíduo.

Em relação ao tabagismo, 14,4\% dos ACS declararam-se fumantes. Esse percentual é superior à média nacional, que é de $9,8 \%$, sendo $12,3 \%$ entre homens e $7,7 \%$ entre as mulheres, ${ }^{22}$ e também superior 
à média de ACS que se declaram fumantes do Brasil (6,6\%). ${ }^{18} \mathrm{O}$ estudo desenvolvido por Andrade ${ }^{23} \mathrm{com}$ 400 ACS em Juiz de Fora/MG observou o uso de tabaco em 10,3\% dos ACS. De acordo com dados da Vigilância de Fatores de Risco para doenças crônicas não transmissíveis - Vigitel, ${ }^{22}$ o Brasil vem apresentando queda no número de fumantes ao longo das décadas, resultado das diversas ações da Política Nacional de Controle do Tabaco. O tabagismo e a exposição passiva ao tabaco são fatores de risco significativos para o desenvolvimento de DCNT, mantendo-se como causas majoritárias de mortes evitáveis em todo o mundo..$^{24,25}$

Acerca do estado nutricional, os resultados do presente trabalho apontaram alta prevalência de sobrepeso e obesidade entre os agentes de saúde avaliados. Esses resultados corroboram os encontrados por Barbosa e Lacerda, ${ }^{26}$ que também avaliaram o estado nutricional de ACS e obtiveram prevalência de $37,4 \%$ de sobrepeso e de $33,7 \%$ de obesidade entre os participantes. Colombo e Derquim, ${ }^{19}$ em estudo realizado com ACS, também observaram prevalência de $51,5 \%$ de excesso de peso entre os participantes da pesquisa.

Nas últimas décadas, tem-se observado aumento significativo no número de pessoas com excesso de peso, o que pode estar relacionado a inúmeros fatores, incluindo baixos índices de atividade física e hábitos alimentares inadequados. É necessário ressaltar que o sobrepeso e a obesidade estão entre os principais fatores de risco para o desenvolvimento de $\mathrm{DCNT},{ }^{22}$ podendo impactar tanto na qualidade de vida quanto no trabalho dos ACS.

No que se refere à distribuição central da gordura corporal, o presente trabalho verificou que a grande maioria dos agentes de saúde avaliados apresentou valores de CC acima dos pontos de corte estabelecidos como adequados. As ACS do sexo feminino apresentaram maior percentual de risco elevado e, sobretudo, risco muito elevado de desenvolver doenças cardiovasculares quando comparadas aos ACS do sexo masculino. Resultados semelhantes foram encontrados por Barbosa e Lacerda, ${ }^{26}$ que verificaram que $20,9 \%$ dos ACS apresentaram risco elevado, e 44,1\% risco muito elevado, para doenças cardiovasculares. Ademais, os autores relataram que há relação entre o sexo e a CC, e que os ACS do sexo feminino apresentaram maiores valores em comparação aos do sexo masculino.

Em relação aos indicadores de adiposidade abdominal, a maior parte dos ACS avaliados apresentou valores elevados de índice de conicidade e RCEst. O acúmulo de gordura na região do abdome está ligado a alterações metabólicas e aumento do risco elevado de morbimortalidade em consequência de aterosclerose e suas complicações, a exemplo da doença arterial coronariana. ${ }^{27} \mathrm{Um}$ estudo realizado por Barroso et al. ${ }^{28}$ apontou relação significativa entre o índice de conicidade e a RCEst, acima dos valores adequados, e a presença de diabetes e hipertensão arterial. A literatura ressalta que a obesidade abdominal está fortemente associada a importantes distúrbios, tais como diabetes tipo II e doenças cardiovasculares. Além disso, já foi demonstrado que o sedentarismo e o tabagismo são alguns dos fatores relacionados a esse tipo de obesidade. ${ }^{29}$

No que diz respeito aos hábitos alimentares, o consumo de alimentos considerados saudáveis, como arroz, feijão, frutas e hortaliças, no dia anterior à entrevista foi relatado pela maioria dos agentes entrevistados. Barbosa e Lacerda ${ }^{26}$ observaram consumo regular de leguminosas e cereais em ACS sem e com excesso de peso. Por outro lado, os autores relataram consumo não regular de frutas e hortaliças em ambos os grupos, sendo o consumo não regular de frutas significativamente maior no grupo de ACS com excesso de peso em comparação aos eutróficos.

No Brasil, 59,7\% da população adulta relata consumo regular de feijão, no entanto, segundo dados da Pesquisa de Orçamentos Familiares, observa-se redução de 12,8\% do consumo dessa leguminosa no 
país. ${ }^{22,30}$ Já o consumo regular de frutas e hortaliças foi referido por apenas $22,9 \%$ dos adultos. ${ }^{22} \mathrm{O}$ Guia Alimentar para a População Brasileira ${ }^{31}$ preconiza que a alimentação seja baseada predominantemente em alimentos in natura ou minimamente processados, como arroz, feijão, frutas e hortaliças. Além disso, a OMS recomenda a ingestão diária de, no mínimo, $400 \mathrm{~g}$ por dia de frutas e hortaliças, o que equivale a cinco porções diárias desses alimentos. ${ }^{32}$

Sabe-se que o aumento na produção e no consumo de AUP é uma das principais causas de obesidade e DCNT na atualidade. ${ }^{33}$ Um estudo realizado pela Organização Pan-Americana de Saúde e pela OMS, na América Latina, observou associação positiva, forte e significativa entre o aumento das

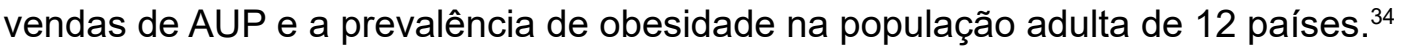

No que diz respeito às limitações do estudo, destaca-se o tipo de instrumento utilizado para a avaliação do consumo alimentar, que pode sofrer influência de viés de memória e que não permite uma avaliação quantitativa no que diz respeito ao tamanho das porções ingeridas e ao perfil de nutrientes. O tamanho reduzido da amostra e o desenho transversal do estudo dificultam a avaliação concreta da relação de causa e efeito entre as variáveis analisadas.

\section{Recomendações para políticas públicas}

Os resultados encontrados no presente trabalho evidenciam a importância da realização de intervenções capazes de promover melhoria nos hábitos de vida dos ACS, sobretudo em relação a sedentarismo, tabagismo, alimentação inadequada e excesso de peso, auxiliando, consequentemente, no enfrentamento das DCNT. ${ }^{35}$ Programas de promoção da saúde do trabalhador devem ser desenvolvidos tanto para o gerenciamento de riscos e doenças quanto para a promoção de hábitos saudáveis e de mudanças no estilo de vida dos trabalhadores. ${ }^{36}$

\section{CONCLUSÕES}

Os achados deste estudo apontam para a elevada prevalência de fatores de risco para o desenvolvimento de DCNT em ACS. No que diz respeito à saúde do trabalhador, o aumento da prevalência dos casos de doenças crônicas pode resultar em absenteísmo e invalidez e repercutir na qualidade do trabalho.

Enquanto profissionais da APS, os agentes comunitários de saúde têm entre suas atividades de rotina o desenvolvimento de atividades relacionadas à promoção da saúde e da alimentação adequada e saudável. Entretanto, por motivos que merecem maior atenção e investigação por parte da gestão, esses hábitos parecem não estar tão presentes no estilo vida dos profissionais.

É importante que a vigilância e a prevenção dos fatores de risco para DCNT, no contexto da saúde do trabalhador, também sejam prioridade no planejamento em saúde dos municípios.

Espera-se que com os resultados deste estudo seja possível melhorar os indicadores de saúde da população estudada e subsidiar a organização de políticas de promoção de saúde, prevenção de doenças e qualidade de vida dos ACS.

\section{CONFLITO DE INTERESSES}

Nada a declarar. 


\section{CONTRIBUIÇÕES DOS AUTORES}

MSB: Administração do projeto, Escrita - primeira redação, Metodologia, Supervisão. JCSC: Conceituação, Escrita - primeira redação, Investigação. RSB: Análise formal, Metodologia, Software, Validação. MHS: Escrita - revisão e edição, Metodologia, Supervisão, Validação. ACS: Conceituação, Escrita - primeira redação, Investigação. MHAT: Administração do projeto, Escrita - revisão e edição, Supervisão, Validação.

\section{REFERÊNCIAS BIBLIOGRÁFICAS}

1. Starfiel B. Atenção primária: equilíbrio entre necessidade de saúde, serviços e tecnologias. Brasília: UNESCO; Ministério da Saúde; 2002.

2. Brasil. Presidência da República. Secretaria-Geral. Subchefia para Assuntos Jurídicos. Lei $n^{\circ} 13.595$, de 5 de janeiro de 2018. Altera a Lei $n^{\circ} 11.350$, de 5 de outubro de 2006, para dispor sobre a reformulação das atribuições, a jornada e as condições de trabalho, o grau de formação profissional, os cursos de formação técnica e continuada e a indenização de transporte dos profissionais Agentes Comunitários de Saúde e Agentes de Combate às Endemias. Brasília; 2018. [acessado em 22 out. 2021]. Disponível em: http://www.planalto.gov.br/ccivil_03/_ato2015-2018/2018/lei/L13595.htm

3. Brasil. Ministério da Saúde. Secretaria de Atenção à Saúde. Departamento de Atenção Básica. O trabalho do agente comunitário de saúde/Ministério da Saúde, Secretaria de Atenção à Saúde, Departamento de Atenção Básica. Brasília: Ministério da Saúde; 2009. [acessado em 22 out. 2021]. Disponível em: http://189.28.128.100/dab/docs/publicacoes/geral/ manual_acs.pdf

4. Brasil. Presidência da República. Secretaria-Geral. Subchefia para Assuntos Jurídicos. Lei $n^{\circ} 13.595$, de 5 de janeiro de 2018. Altera a Lei $n^{\circ} 11.350$, de 5 de outubro de 2006, para dispor sobre a reformulação das atribuições, a jornada e as condições de trabalho, o grau de formação profissional, os cursos de formação técnica e continuada e a indenização de transporte dos profissionais Agentes Comunitários de Saúde e Agentes de Combate às Endemias [Internet] 2018. [acessado em 08 nov 2021]. Disponível em: http://www.planalto.gov.br/ccivil_03/_ato2015-2018/2018/lei/L13595.htm

5. Heloani JR, Capitão CG. Saúde mental e psicologia do trabalho. São Paulo em Perspectiva. 2003;17(2):102-8. https://doi. org/10.1590/S0102-88392003000200011

6. Baralhas M, Pereira MAO. Prática diária dos agentes comunitários de saúde: dificuldades e limitações da assistência. Rev Bras Enferm 2013;66(3):358-65. https://doi.org/10.1590/S0034-71672013000300009

7. Costa MC, Silva EB, Jahn AC, Resta DG, Colom ICS, Carli R. Processo de trabalho dos agentes comunitários de saúde: possibilidades e limites. Rev Gaúcha Enferm 2012;33(3):134-40. https://doi.org/10.1590/S1983-14472012000300018

8. Souza EB. Transição nutricional no Brasil: análise dos principais fatores. Cadernos UniFOA 2010;5(13):49-53. https://doi. org/10.47385/cadunifoa.v5i13.1025

9. World Health Organization. Obesity: preventing and managing the global epidemic. Geneva: World Health Organization; 1998.

10. Organización Panamericana de la Salud. División de Promoción y Protección de la Salud (HPP). Encuesta multicentrica salud bienestar y envejecimiento (SABE) en América Latina y el Caribe. Washington: Organización Panamericana de la Salud; 2001.

11. World Health Organization. Waist circumference and waist-hip ratio. Geneva: World Health Organization; 2008.

12. Pitanga FJG, Lessa I. Indicadores antropométricos de obesidade como instrumento de triagem para risco coronariano elevado em adultos na cidade de Salvador - Bahia. Arq Bras Cardiol 2005;85(1):26-31. https://doi.org/10.1590/S0066782X2005001400006

13. Pitanga FJG, Lessa I. Razão cintura-estatura como discriminador do risco coronariano de adultos. Rev Assoc Med Bras 2006;52(3):157-61. https://doi.org/10.1590/S0104-42302006000300016

14. Brasil. Ministério da Saúde. Secretaria de Atenção à Saúde. Departamento de Atenção Básica. Orientações para avaliação de marcadores de consumo alimentar na atenção básica [recurso eletrônico]/Ministério da Saúde, Secretaria de Atenção à Saúde, Departamento de Atenção Básica. Brasília: Ministério da Saúde; 2015. Disponível em: https://bvsms.saude.gov.br/ bvs/publicacoes/marcadores_consumo_alimentar_atencao_basica.pdf

15. Craig CL, Marchall AL, Sjostrom M, Baumam AE, Booth ML, Ainsworthh BE, et al. International physical activity questionnaire: 12-country reability and validity. Med Sci Sports Exerc 2003;35(8):1381-95. https://doi.org/10.1249/01. MSS.0000078924.61453.FB

16. Bezerra AFB, Espirito Santo ACG, Batista Filho M. Concepções e práticas do agente comunitário na atenção à saúde do idoso. Rev Saude Publica 2005;39(5):809-15. https://doi.org/10.1590/S0034-89102005000500017

17. Machado MH. Trabalhadores da saúde e sua trajetória na reforma sanitária. In: Cadernos RH Saúde/Ministério da Saúde, Secretaria de Gestão do Trabalho e da Educação na Saúde. Brasília: Ministério da Saúde; 2006. p. 13-28. Disponível em: https://bvsms.saude.gov.br/bvs/publicacoes/cadernos_rh.pdf 
18. Paim JS. Análise de políticas de saúde no Brasil (2003-2017): relatório final, 2018. Salvador: Universidade Federal da Bahia; 2018. Disponível em: https://analisepoliticaemsaude.org/oaps/documentos/boletim/relatorio-do-cnpq2018/

19. Colombo PAR, Derquin ES. Avaliação do perfil antropométrico (índice de massa corporal - IMC), hábitos alimentares e atividade física, em Agentes Comunitárias de Saúde (ACS), do município de Colider-MT. Revista Brasileira de Obesidade, Nutrição e Emagrecimento 2008;2(11):490-97.

20. Moura EC, Morais Neto OL, Malta DC, Moura L, Silva NN, Bernal R, et al. Vigilância de fatores de risco para doenças crônicas por inquérito telefônico nas capitais dos 26 estados brasileiros e no Distrito Federal (2006). Rev Bras Epidemiol 2008;11(supl 1):20-37. https://doi.org/10.1590/S1415-790X2008000500003

21. Maia EG, Gomes FMD, Alves MH, Huth YR, Claro RM. Hábito de assistir à televisão e sua relação com a alimentação: resultados do período de 2006 a 2014 em capitais brasileiras. Cad Saúde Pública 2016;32(9):e00104515. https://doi. org/10.1590/0102-311X00104515

22. Brasil. Ministério da Saúde. Secretaria de Vigilância em Saúde. Departamento de Análise em Saúde e Vigilância de Doenças Não Transmissíveis. Vigitel Brasil 2019: vigilância de fatores de risco e proteção para doenças crônicas por inquérito telefônico: estimativas sobre frequência e distribuição sociodemográfica de fatores de risco e proteção para doenças crônicas nas capitais dos 26 estados brasileiros e no Distrito Federal em 2019 [recurso eletrônico]/Ministério da Saúde, Secretaria de Vigilância em Saúde, Departamento de Análise em Saúde e Vigilância de Doenças não Transmissíveis. Brasília: Ministério da Saúde; 2020. Disponível em: https://bvsms.saude.gov.br/bvs/publicacoes/vigitel_brasil_2019_vigilancia_fatores_risco. pdf

23. Andrade CCB. A hipertensão arterial em agentes comunitários de saúde [dissertação de mestrado]. Juiz de Fora: Faculdade de Enfermagem da Universidade Federal de Juiz de Fora; 2017.

24. World Health Organization. WHO Framework Convention on Tobacco Control. History of the WHO framework convention on tobacco control. Geneva: World Health Organization; 2009. [acessado em 22 out. 2021]. Disponível em: https://apps.who. int/iris/bitstream/handle/10665/44244/9789241563925_eng.pdf

25. World Health Organization. WHO report on the global tobacco epidemic, 2011: warning about the dangers of tobacco. Geneva: World Health Organization; 2011. [acessado em 22 out. 2021]. Disponível em: https://apps.who.int/iris/bitstream/ handle/10665/44616/9789240687813_eng.pdf?sequence=1\&isAllowed=y

26. Barbosa AM, Lacerda DAL. Associação entre consumo alimentar e estado nutricional em agentes comunitários de Saúde. Rev Bras Ci Saúde 2017;21(3):189-96.

27. Almeida RT, Almeida MMG, Araújo TM. Obesidade abdominal e risco cardiovascular: desempenho de indicadores antropométricos em mulheres. Arq Bras Cardiol 2009;92(5):375-80. https://doi.org/10.1590/S0066-782X2009000500007

28. Barroso TA, Marins LB, Alves R, Gonlçalves AS, Barroso SG, Rocha GS. Associação entre a obesidade central e a incidência de doenças e fatores de risco cardiovascular. Int J Cardiovasc Sci 2017;30(5):416-24. https://doi.org/10.5935/23594802.20170073

29. Sabóia RS, Araújo AP, Barbosa JMA, Galvão CEP, Cruvel, JMS, Ferreira SCN. Obesidade abdominal e fatores associados em adultos atendidos em uma clínica escola. Rev Bras Promoç Saúde 2016;29(2):259-67.

30. Instituto Brasileiro de Geografia e Estatística. Coordenação de Trabalho e Rendimento. Pesquisa de orçamentos familiares 2017-2018: primeiros resultados. Rio de Janeiro: Instituto Brasileiro de Geografia e Estatística; 2019. Disponível em: https:// biblioteca.ibge.gov.br/visualizacao/livros/liv101670.pdf.

31. Brasil. Ministério da Saúde. Secretaria de Atenção à Saúde. Departamento de Atenção Básica. Guia alimentar para a população brasileira/Ministério da Saúde, Secretaria de Atenção à Saúde, Departamento de Atenção Básica. $2^{a}$ ed. Brasília: Ministério da Saúde; 2014. [acessado em 22 out. 2021]. Disponível em: https://bvsms.saude.gov.br/bvs/publicacoes/guia_ alimentar_populacao_brasileira_2ed.pdf

32. World Health Organization. Fruit and vegetable promotion initiative: a meeting report. Geneva: World Health Organization; 2003. [acessado em 22 out. 2021]. Disponível em: https://apps.who.int/iris/bitstream/handle/10665/68395/WHO_NMH_ $\mathrm{NPH}$ NNP_0308.pdf?sequence=1\&isAllowed=y

33. Monteiro CA, Levy RB, Claro RM, Castro IRR, Cannon G. A new classification of foods based on the extent and purpose of their processing. Cad Saude Publica 2010;26(11):2039-49. https://doi.org/10.1590/s0102-311x2010001100005

34. Pan American Health Organization. Ultra-processed food and drink products in Latin America: trends, impact on obesity, policy implications. Washington: Pan American Health Organization; 2015. [acessado em 22 out. 2021]. Disponível em: https://iris.paho.org/bitstream/handle/10665.2/7699/9789275118641_eng.pdf

35. Brasil. Ministério da Saúde. Secretaria de Vigilância em Saúde. Dēpartamento de Vigilância de Doenças e Agravos não Transmissíveis e Promoção da Saúde. Relatório do III Fórum de Monitoramento do Plano de Ações Estratégicas para o Enfrentamento das Doenças Crônicas não Transmissíveis no Brasil [recurso eletrônico]/Ministério da Saúde, Secretaria de Vigilância em Saúde, Departamento de Vigilância de Doenças e Agravos não Transmissíveis e Promoção da Saúde. Brasília: Ministério da Saúde; 2018. [acessado em 22 out. 2021]. Disponível em: https://bvsms.saude.gov.br/bvs/publicacoes/ relatorio_iii_forum_monitoramento_plano.pdf

36. Sousa-Uva A, Serranheira F. Trabalho e saúde/doença: o desafio sistemático da prevenção dos riscos profissionais e o esquecimento reiterado da promoção da saúde. Rev Bras Med Trab 2013;11(1):43-9. 